\title{
Etiological Profile of Atrial Fibrillation (AF) In Nepalgunj Medical College: A Hospital Based Study
}

Kidwai A

\begin{abstract}
Introduction: Atrial fibrillation (AF) is the commonest cardiac arrhythmia encountered in clinical practice. The hall mark sign of atrial fibrillation is an irregular rhythm in ECG with no obvious P wave. In Western countries, Coronary Artery Disease (CAD) is the commonest cause of AF. Plenty of data and studies are available regarding the epidemiology and etiology of AF in the Western Population but similar studies and data in Nepal are scarce. This study was therefore conducted with an objective to establish the etiological profile of atrial fibrillation patients in mid western Nepal. Materials and Methods: This was a hospital based study, carried out in the Department of Internal Medicine, Nepalgunj Medical College Teaching Hospital, Nepalgunj, for a duration of 1 year from $14^{\text {th }}$ September 2013 to $13^{\text {th }}$ September 2014. A total of 74 consecutive cases diagnosed as AF were included in the study on the basis of inclusion and exclusion criteria. Results: $A$ total of 74 consecutive patients were included in the study. The mean age of the subjects was 39.2 years. The majority of the subjects were below 50 years of age (70.27\%). There were only 2 subjects below 19 and 3 patients above 70 years of age. Out of the 74 patients 52(70.2\%) were from the age group of below 50 years of age. In western countries the incidence is higher in the elder population. Rheumatic heart disease was the most common etiology in this study with a total of $29(39.2 \%)$ cases. CAD in our study was seen in 12 patients (16.2\%). Overall structural heart disease was seen in 64(86.5\%) cases and in the rest of the cases echocardiography was normal. Conclusion: This study has shown that the etiological profile of $A F$ is different in patients attending our hospital situated in mid-western Nepal from western countries. Unlike the western countries AF is more common in the younger age group and the most common etiology in Nepal is RHD, whereas in western countries it is CAD.
\end{abstract}

Key words: Atrial Fibrillation (AF), Rheumatic heart disease (RHD), Coronary Artery Disease (CAD)

\section{INTRODUCTION}

Atrial fibrillation (AF) is the commonest cardiac arrhythmia encountered in clinical practice ${ }^{1}$. It is characterized by irregular, disorganized and chaotic electrical activity of the atrium. It is an important cause of morbidity and mortality not only in the western world but in Nepal as well. AF is found in $0.4 \%$ adults below 60 years of age. Incidence of AF appears to double with each decade. In the 9th decade the incidence is thought to be as high as $8-10 \%^{2}$. Clinically, it is recognized by irregularly irregular pulse with varying rates ranging from normal to 200 and a pulse deficit of more than 10 beats per minute. The hall mark sign of atrial fibrillation is an irregular rhythm in ECG with no obvious $P$ wave. Established risk factors for AF include cardiac conditions, such as systolic and diastolic heart failure, valvular heart disease, and myocardial infarction, and cardiovascular risk factors, such as hypertension, diabetes mellitus, obesity, and cigarette smoking ${ }^{3,4}$. In Western countries, CAD is the commonest cause of $\mathrm{AF}^{5}$. Atrial fibrillation has been classified by American Heart Association/ American college of cardiology/European Society of cardiology into first detected episode, recurrent (two or more episode), paroxysmal

Address for correspondence:

Dr Aasim Kidwai

Department of Medicine

Nepalgunj Medical College

Nepalgunj, Banke, Nepal

Email: kidwai.aasim@gmail.com (terminates within 7 days), persistent (persist for more than 7 days) and permanent (sustained for more than 1 year or has failed cardio version) $)^{6,7}$.

Clinical presentation can vary from being asymptomatic, to atrial fibrillation (AF) with rapid ventricular response to cardiogenic shock or devastating cerebrovascular accident (CVA). Although up to $90 \%$ of AF episodes may not cause symptoms, many patients experience a wide variety of symptoms, including palpitations, dyspnea, fatigue, dizziness, angina, and decompensated heart failure. In addition, AF can be associated with hemodynamic dysfunction, tachycardiainduced cardiomyopathy and systemic thromboembolism ${ }^{8}$.

Plenty of data and studies are available regarding the etiology of AF in the western population but similar studies and data in Nepal is scarce. Only a few studies have been done and most of them have been conducted in eastern and central Nepal. This study was therefore conducted with an objective to establish the etiological profile of atrial fibrillation patients in mid western Nepal.

\section{MATERIALS AND METHODS}

This was an observational study which was carried out at the Department of Internal Medicine, Nepalgunj Medical College Teaching Hospital, Nepalgunj, for a duration of 1 year from $14^{\text {th }}$ September 2013 to 13th September 2014. A total of 74 consecutive cases diagnosed as AF were included in the study on the basis of inclusion and exclusion criteria. The diagnosis of 
AF was made on the basis of history, clinical examination, examination of previous ECGs and confirmation with 12 leads ECG.

Inclusion criteria

1. Patients who give informed consent

2. History of atrial fibrillation on the basis of previous documentation and clinical examination

3. Irregularly irregular pulse rate

4. ECG findings: Absence of well-defined $P$ waves, irregularly irregular rhythm and absence of isoelectric basline.

Exclusion criteria

1. Patients who do not give consent.

2. ECG findings: evidence of other arrhythmias ( atrial flutter, heart blocks, ventricular arrhythmias, junctional arrhythmias )
All the confirmed cases were thoroughly evaluated for the possible etiology by history, clinical examination, Trans Thoracic Echocardiography, Thyroid function test, serum electrolytes, chest xrays, Haemogram, liver function tests, renal function tests at other relevant laboratory investigations. Patients were considered as having structural heart disease if any abnormality was detected in the trans-thoracic echocardiography. Data analysis was done using Microsoft Excel 2013

\section{RESULTS}

A total of 74 consecutive patients were included in the study. The age distribution was as follows. The mean age of the subjects was 39.2 years. The majority of the subjects were below 50 years of age (70.27\%). There were only 2 subjects below 19 and 3 patients above 70 years of age.

\begin{tabular}{|l|c|c|}
\hline Age group ( years ) & Number of Subjects & Percentage \\
\hline$<19$ & 2 & $2.7 \%$ \\
\hline $19-29$ & 20 & $27.0 \%$ \\
\hline $30-39$ & 18 & $24.3 \%$ \\
\hline $40-49$ & 12 & $16.2 \%$ \\
\hline $50-59$ & 10 & $13.5 \%$ \\
\hline $60-70$ & 9 & $12.2 \%$ \\
\hline$>70$ & 3 & $4.1 \%$ \\
\hline
\end{tabular}

Table I: Age distribution

\begin{tabular}{|l|c|c|}
\hline Sex & No. of patients & Percentage \\
\hline Female & 33 & $44.6 \%$ \\
\hline Male & 41 & $55.4 \%$ \\
\hline
\end{tabular}

Table II: Sex distribution

\begin{tabular}{|l|c|c|}
\hline Etiology & No. of cases & Percentage \\
\hline RHD : Pure Mitral Valve Disease & 19 & $25.6 \%$ \\
\hline RHD : Mitral + Aortic Valve Disease & 10 & $13.5 \%$ \\
\hline Non Rheumatic Valvular Heart Disease & 6 & $8.2 \%$ \\
\hline Coronary Artery Disease & 12 & $16.2 \%$ \\
\hline Hypertensive Heart Disease & 7 & $9.5 \%$ \\
\hline Cardiomyopathy & 5 & $6.7 \%$ \\
\hline Hyperthyroidism & 3 & $4.1 \%$ \\
\hline COPD With COR Pulmonale & 5 & $6.7 \%$ \\
\hline COPD Without COR Pulmonale & 3 & $4.0 \%$ \\
\hline ONLY Diabetes Mellitus & 1 & $1.4 \%$ \\
\hline UNKNOWN ( LONE AF ) & 3 & $4.1 \%$ \\
\hline
\end{tabular}

Table III: Etiology of Atrial Fibrillation 


\section{DISCUSSION}

This study showed a different pattern of etiology in patients of AF compared to the western countries. Out of the 74 patients, $52(70.2 \%)$ were from the age group of below 50 years of age, whereas in western countries the incidence is higher in the elderly (>60years) population ${ }^{2,9,10}$. However this data is similar to the data reported by MP Gautam et $\mathrm{al}^{11}$ in their study of AF in patients from central Nepal, which showed that most of the subjects were comparatively of a younger age group than reported in most of the studies in the western population. Our study also has shown a similar result in the mid-western Nepalese population. $41(55.4 \%)$ patients were male and 33 $(44.6 \%)$ were female which is similar to The Framingham Heart study ${ }^{2}$.

Rheumatic heart disease was the most prevalent etiology in the study with a total of 29 (39.2\%) cases. This data is similar to the study done by MP Gautam et al which also showed RHD as the commonest etiology. ${ }^{11}$ Similar findings were also reported by $\mathrm{A}$ $V{ }^{12}{ }^{12}$ and also by $M$ Dhakal et $\mathrm{al}^{13}$ in their study which showed RHD as the most prevalent etiology in India. These data are different from the data done in the western population, where the commonest etiology in most of studies was $C A D^{2,9,10}$. CAD in our study was seen in 12 patients (16.2\%) and was the second most common etiology after RHD. Pure mitral valve disease was seen in $19(25.6 \%)$ patients.

Overall 64 (86.5\%) cases had abnormal echocardiograms and were labelled as having structural heart disease. COPD was seen in 8 cases and all of the patient were over 60 years of age. Echocardiography showed dilated right ventricle and atrium in 5 cases and therefore they were included in the structural heart disease group and 3 patients had normal echocardiography. Cardiomyopathy was seen in 5 cases and all had dilated ventricle with reduced ejection fraction $(<50 \%)$. Diabetes was present in 12 cases but it was associated with structural heart disease except in 1 case in which no other etiology was detected. Hypertensive heart disease was seen in 7 cases and non-rheumatic valvular disease was seen in 6 cases. Hyperthyroidism was detected in 3 patients. No etiology could be found in 3 patients and these patients were labeled as lone AF.

\section{CONCLUSION}

This study has shown that the etiological profile of patients with AF in mid-western Nepal which is different from that of western countries $^{2,9,10}$, is similar to that of central Nepal ${ }^{11}$ and also of India ${ }^{12,13}$. Unlike the western countries where CAD is the commonest etiology of $\mathrm{AF}$ and $\mathrm{AF}$ is more prevalent in the elder age group, in mid western Nepal RHD is the most common cause. RHD is primarily a disease of the young ${ }^{5}$ and this would explain the higher prevalence of $A F$ in younger age group in Nepal compared to western countries. The risk factors for RHD are overcrowding, poor hygiene and sanitation and therefore it is considered as a disease of the developing nations ${ }^{14,15}$. This might have contributed to the high prevalence of AF in RHD and in younger age groups in Nepal, as it is a developing nation and the data of studies done in other developing nations like India also show a similar pattern ${ }^{12,13}$.

As data regarding epidemiology and etiology of AF in the Nepalese population is scarce, this study showed us the difference in the profile of the patients in Nepal from the western countries. This could help us in the management of AF and the underlying pathology by keeping an open mind about the common causes in our region. However more detailed epidemiologic studies are required to improve the understanding of the burden of AF in Nepal.

\section{REFERENCE}

1. Nand Vidya et al. Etiological profile and clinical presentation of patients with atrial fibrillation from a rural area in Bihar. National journal of medical research, 2012;2:2:124.

2. Kannel WB, Abbot RD, Savage DD, McNemara PM. Epidemiologic features of chronic AF. The Framingham Heart study. N Engl J Med, 1982; 307:1018-1022

3. Benjamin EJ, Levy D, Vaziri SM et al. Independent risk factors for atrial fibrillation in a population-based cohort: the Framingham Heart Study. JAMA. 1994; 271: 840-4

4. Thomas MC, Dublin S, Kaplan RC et al. Blood pressure control and risk of incident atrial fibrillation. Am J Hypertens. 2008; 21: 1111-6.

5. Lip GYH, Beevers DG. History, epidemiology and importance of atrial fibrillation. BMJ. 1995; 311(7016): 1361-3.

6. Fuster V, Ryden LE, Asinger RW et al. ACC/AHA/ESC guidelines for the management of patient with atrial fibrillation (Committee to develop guidelines for the management of patients with $\mathrm{AF}$ ). Circulation 2001; 104: 2118-50.

7. Levy S. Classification system of Atrial Fibrillation. Cur. Opin. Cardiol. 2000;15:54-7.

8. Page RL etal. Asymptomatic arrhythmias in patients with symptomatic paroxysmal atrial fibrillation and paroxysmal supraventricular tachycardia. Circulation. 1994;89(1):224-7.

9. Thomas Wilke, Antje Groth, Sabrina Mueller et al. Incidence and Prevalence of Atrial Fibrillation. An Analysis Based on 8.3 Million Patients Europace. 2013; 15(4):486-493.

10. Guize L, Thomas F, Bean K, Benetos A, Pannier B. Atrial fibrillation. Prevalence, risk factors and mortality in a large French population with 15 years of follow-up. Bull Acad 2007; Apr-May 191(45):791-803.

11. MP Gautam etal. A study of the clinical profile of atrial fibrillation in a tertiary care super-specialty referral centre in Central Nepal. Journal of College of Medical Sciences-Nepal, 2012. 8; 3: 9-16.

12. A Vora. Approach to Management of Atrial Fibrillation in the Indian Scenario SUPPLEMENT OF JAPI. APRIL 2007;55:20-23.

13. M Dhakal etal. Epidemiology and clinical presentation of patients with atrial fibrillation from a tertiary care hospital of east Sikkin: an observational study. JEMDS 2013; 2: 20: 3554-3560.

14. Community control of rheumatic heart disease in developing countries: 1 a major public health problem. WHO Chron. 1980;34:336-45

15. Agarwal BL. Rheumatic heart disease unabated in developing countries. Lancet. 1981;2:910-1. 